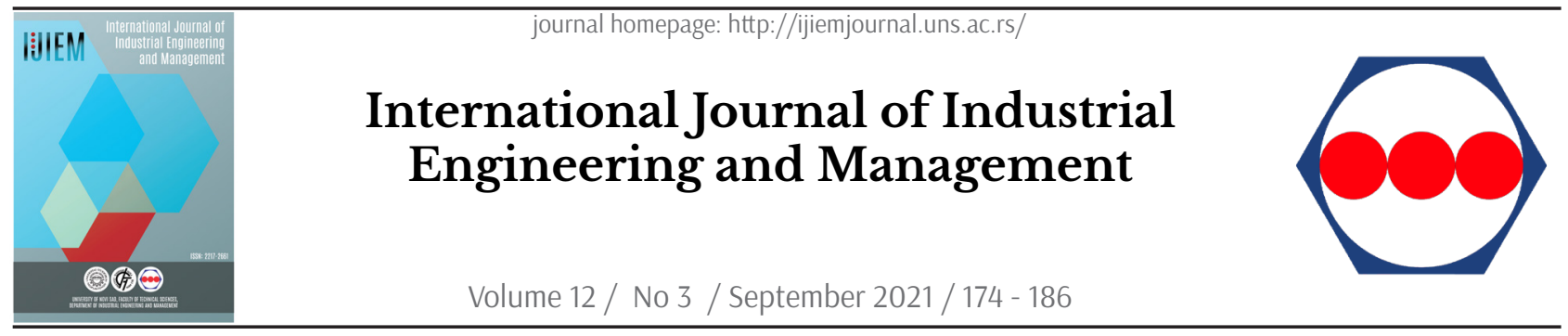

Original research article

\title{
Layout and scheduling optimization problem for a reconfigurable manufacturing system
}

\author{
S. Gao*, J. Daaboul , J. Le Duigou \\ Université de technologie de Compiègne, CNRS, Roberval (Mechanics energy and electricity), \\ Centre de recherche Royallieu - CS 60319 - 60203 Compiègne Cedex, France
}

\section{A B STRACT}

Increased product variety leads manufacturers to adapt more flexible manufacturing systems such as the reconfigurable manufacturing system. This paper establishes a linear mathematical model to minimize the tardiness penalty for job shop scheduling and layout optimization in a Reconfigurable Manufacturing System. A small example solved by CPLEX software is used as a first step to validate the mathematical model. An improved Genetic Algorithm approach is introduced to search the approximate optimal solutions of this model in an efficient way and is tested using numerical experiments.

\section{ARTICLE INFO}

Article history:

Received January 14, 2021

Revised June 30, 2021

Accepted July 2, 2021

Published online September 8, 2021

Keywords:

Scheduling;

Layout;

Reconfigurable manufacturing

system;

Machine reconfiguration;

*Corresponding author:

Sini Gao

sini.gao@utc.fr

\section{Introduction}

Nowadays, more and more people expect to purchase customized goods of great quality at a reasonable price, which asks manufacturers to supply products not only at low cost and with high quality but also in a great variety [1]. Mass Customization (MC) is a modern production paradigm for producing customized products at about the same cost as mass production [2]. It requires flexibility in the manufacturing system [3]. The Dedicated Manufacturing Line (DML) is cost-effective around one specific product [4]. Nevertheless, its configuration is fixed. Flexible Manufacturing System (FMS) offers general flexibility for a wide range of parts variants, but the equipment is expensive and productivity is low. Therefore, many researchers from industry or academia are trying to develop the Reconfigurable Manufacturing System (RMS), which combines the high yields of DML with the flexibility of FMS [5].

RMS is defined as a system designed at the outset for rapid change in structure, as well as in hardware and software components, in order to quickly adjust production capacity and functionality within a part family in response to sudden changes in the market or regulatory requirements [6]. Reconfiguration in RMS divides into machine-level reconfiguration and system-level reconfiguration [7]. Changes such 
as moving, adding, and removing equipment are considered system-wide reconfiguration. Changes to machine configuration or Reconfigurable Manufacturing Tool (RMT) are regarded as reconfiguration at the machine level [8].

Both reconfiguration levels impact system performance, hence should be both optimized. Reconfiguration at the machine level will probably extend the makespan, even with optimized scheduling, yet the layout reconfiguration will likely shorten the duration of Work-in-Progress (WIP) transport between separate machines. Thus, performing as many operations as possible on a machine installed by a selected configuration improves efficiency. Optimized layout is significant in minimizing the overall completion time. However, disparate due dates and weights for tardiness penalty complicate these principles in the scheduling problem. Apart from machine location, the layout issue has to consider machine shape. Since they are closely dependent, layout optimization and scheduling should be simultaneously considered to improve efficiency.

This paper attempts to simultaneously optimize the tardiness for jobs in RMS by integrating the jobshop scheduling and layout problem. It proposes a mathematical model for the integrated problem and validates it using CPLEX and then proposes a Genetic Algorithm (GA) based approach for its resolution. It is organized as follows: the literature review is presented in Section 2; the mathematical model to formulate the problem is explained in Section 3; the numerical experiments to validate the improved heuristic approach are introduced in Section 4; the computational results are discussed in Section 5; the conclusion about limitations and future work is summarized in Section 6.

\section{Literature review}

The literature review examined two keywords pairs 'reconfigurable manufacturing system' with 'scheduling' and 'reconfigurable manufacturing system' with 'layout' in four databases, which are Elsevier (sciencedirect.com), Springer (springerlink.com), IEEE (ieeexplore.ieee.org), and Taylor \& Francis (tandfonline.com).

Research on scheduling in RMS mainly embraces the job shop theory to optimize the completion time in the mixed-integer model. Li et al. [9] presented a mathematical approach for distributing the stochastic demands and exchanging machines or modules among lines (which are groups of machines) for adaptively configuring these lines and machines for the resulting shared demand under a limited inventory of configurable components. Mahmoodjanloo et al. [10] dealt with a flexible job shop scheduling problem with RMTs by formulating two mixed-integer linear programming models with the position-based and sequence-based decision variables to minimize the maximum completion time. Roshanaei et al. [11] solved both partially and totally flexible job shop scheduling problem by developing two novel effective position-based and sequence-based mixed integer linear programming (MILP) models to minimize the maximum completion time. Azab et al. [12] formulated a mixed-integer linear programming model considering both family sequencing and operations sequencing inside each family. Abbasi et al. [13] developed a mixed integer nonlinear programming model to determine optimum sequence of production tasks, corresponding configurations, and batch sizes. Bhargav et al.[14] built an entire integer model to minimize the makespan of the product by segregating and scheduling the similar operations of product in RMS. Genetic algorithm is widely adopted to handle the related problem. Aiping et al. [15] introduced an two-objectives optimization model to achieve the robust scheduling by a genetic algorithm (GA) embedded with extended timed-place petri nets (ETPN). Ladosz et al. [16] presented a genetic algorithm used for dynamic product routing in RMS. Ye et al. [17] put forward a genetic algorithm (GA) with parallel chromosome coding scheme to solve the integrated modular product scheduling and manufacturing cell configuration problem in RMSs. Recently, some researchers integrate scheduling with other issues. Dou et al. [18] integrated optimization problem of configuration design and scheduling for RMS by presenting a multi-objective particle swarm optimization (MoPSO) based on crowding distance and external Pareto solution archive. Khezri et al. [19] proposed a multi-objective scheduling model to optimize the processing time and cost in RMS as well as the emission of liquid hazardous waste and greenhouse gas emissions (GHG).

Maganha et al. [20] presented a literature review about the layout design in RMS and classified this problem into the choices of machines and the layout of the manufacturing system. The choices of machines decide the facilities allocated in the layout. Klement et al. [21] considered a two-objectives model to allocate a number of identical mobile robots to the workstations. Meccanica [22] adopted a negotiation model for solving the problem of allocating production plants to product groups without specific location 
information. The layout of the manufacturing system dominates the particular location of machines. Haddou et al. [23] proposed two-phase-based approach combining the well-known metaheuristic, archived multi-objective simulated annealing (AMOSA), with an exhaustive search-based heuristic to determine the best machine layout for all the selected machines of the product family. Wei et al. [24] proposed a chaotic genetic algorithm with improved Tent mapping to solve the problems associated with the organization of the dynamic facility layout in RMS. Sui et al. [25] established a mathematical model of the equipment layout in the RMS workshop and designed the fitness function with penalty factor which is based on the minimum principle of logistics cost and the physical constraints of the workshop layout. Yamada et al. [26] combined these two aspects by proposing a layout optimization method for manufacturing cells and an allocation optimization method for transportation robots in RMSs was solved by using a particle swarm optimization method.

From above, multi-objective mathematical models and meta-heuristic algorithms are the most used methods in this research area. Researchers prefer to employ integer variables deciding the operation processing order, giving rise to a large number of decision variables. Up to our knowledge, there is no work jointly optimizing layout and scheduling in RMS, yet the layout impacts scheduling decisions and overall system efficiency. Also, RMS aim is to be able to quickly reconfigure, hence reconfiguration at system level including layout design should be considered. Less research on layout design considers an open field than that among available locations. Hence, this paper aims to join an open-field layout design in a flat workshop with the scheduling in an RMS. The beginning time of each operation and the machine location are specified as independent continuous decision variables. The processing sequence is implicit in the values of these decision variables.

\section{Mathematical model}

\subsection{Problem statement}

A product is composed of a number of parts. A job represents the procedure to accomplish the entire processing of a part. Let us consider that there are $n$ jobs. Each job consists of several operations. An operation is an activity to create a feature of a part, such as drilling a hole. Activities to create the same feature with distinct attributes are thought as the same operation. Same operations may take distinct processing time, like drilling holes in different diameters. An operation may be required for more than one job. It is assumed that one operation could appear just once in a job and the operation sequence for every job is determined. There are p operations in total.

Machines in RMS have several configurations each. In this paper, each configuration is assumed to process only one operation and each operation is assumed to be carried out merely on one configuration. Hence, the feasible machine for each operation is identified. The reconfiguration time between different configurations is perceived as that between different operations.

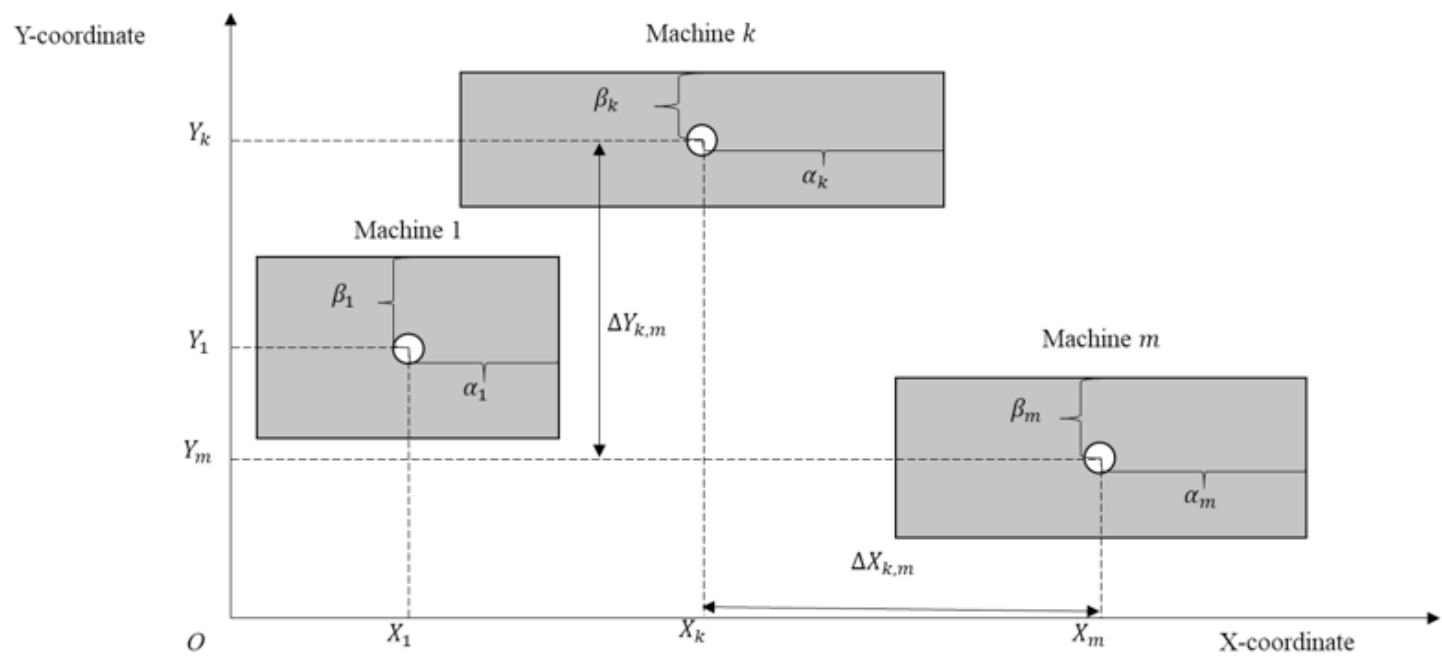

Figure 1.The open-field layout in an RMS 
To simplify the calculation in WIPs transportation, the time to move every WIP in the unit distance is a constant of 1 . Movements are supposed to take place exclusively in the horizon and vertical directions. Consequently, the value of a WIP transportation time is equal to the distance between machines. The sum of the distance between the two machines on the $\mathrm{X}$-coordinate and the $\mathrm{Y}$-coordinate represents the distance between them. As shown in Figure 1, the distance between Machine $k$ and Machine $m$ is equal to the distance between Machine $k$ and Machine $m$ on the $\mathrm{X}$-coordinate $\Delta X_{k, m}$ added to the distance between Machine $k$ and Machine $m$ on the Y-coordinate $\Delta Y_{k, m}$. All machines have a rectangular security area, whose scopes are estimated by their shape of length and width. To prevent the overlap of these areas, the location intervals should respect the limits of the security distances

\subsection{Mathematical model}

The indexes are as follows:

$i, i$ ' Index for jobs, $\forall \mathrm{i}, \mathrm{i}^{\prime} \in\{1,2, \ldots, \mathrm{n}\}$

$e, e$ ' Index for operations, $\forall \mathrm{e}, \mathrm{e}^{\prime} \in\{1,2, \ldots, \mathrm{p}\}$

$j, j$ ' Index for position in the operation sequence, $\forall j, j, \in\left\{1,2, \ldots,\left|\rho_{\mathrm{i}}\right|\right\}$

$k, k^{\prime}$ Index for machines, $\forall \mathrm{k}, \mathrm{k}^{\prime} \in\{1,2, \ldots, \mathrm{m}\}$

The parameters are as follows: $n \quad$ Number of jobs

$p \quad$ Number of operations

$m$ Number of machines

$\rho_{i, j}$ Corresponding operation at the $j^{t h}$ position in the operation sequence of job $i$

$\varphi_{e} \quad$ Corresponding machine for operation $e$

$t_{i, e} \quad$ Processing time for operation $e$ from job $i$

$r_{e, e}$, Reconfiguration time from operation $e$ to operation $e^{\prime}$, if $e=e^{\prime}, r_{e, e^{\prime}}=0$

$d_{i} \quad$ Due date of job i

$W_{i}$ The penalty for unit tardiness time of job $i$

$\alpha_{\mathrm{k}}$ The security distance for machine $k$ on the $\mathrm{X}$-coordinate

$\beta_{k}$ The security distance for machine $k$ on the Y-coordinate

This linear mathematical model aims to minimize the penalty for all the jobs caused by tardiness. Coefficients of tardiness penalty are used and they indicate the priority to process these parts. The objective function is:

$$
\text { Minimize } \sum_{i=1}^{n} T_{i} \times W_{i}
$$

The dimension of all the parameters and variables are 1 . The decision variables are as follows:

$B_{i, j}$ Continuous variable for the beginning time of the jth operation in the operation

$$
\begin{aligned}
& C_{i, j}=B_{i, j}+t_{i, \rho_{i, j}} \\
& \forall i \in\{1,2, \ldots, n\}, \forall j \in\left\{1,2, \ldots,\left|\rho_{i}\right|\right\}(1) \\
& T_{i}=\max \left(C_{i,\left|\rho_{i}\right|}-d_{i}, 0\right) \quad \forall i \in\{1,2, \ldots, n\}(2) \\
& \Delta X_{k, k^{\prime}}=\left|X_{k}-X_{k^{\prime}}\right| \quad \forall k, k^{\prime} \in\{1,2, \ldots, m\}(3) \\
& \Delta Y_{k, k^{\prime}}=\left|Y_{k}-Y_{k^{\prime}}\right| \quad \forall k, k^{\prime} \in\{1,2, \ldots, m\} \text { (4) } \\
& \Delta X_{k, k^{\prime}} \geq \alpha_{k}+\alpha_{k^{\prime}} \| \Delta Y_{k, k^{\prime}} \geq \beta_{k}+\beta_{k^{\prime}} \\
& \forall k, k^{\prime} \in\{1,2, \ldots, m\}(5) \\
& B_{i, j+1} \geq C_{i, j}+r_{\rho_{i, j}, \rho_{i, j+1}}+\Delta X_{\varphi_{\rho_{i, j}} \varphi_{\rho_{i, j+1}}}+\Delta Y_{\varphi_{\rho_{i, j}} \boldsymbol{\rho}_{\rho_{i, j+1}}} \\
& \forall i \in\{1,2, \ldots, n\}, \forall j \in\left\{1,2, \ldots,\left|\rho_{i}\right|-1\right\}(6) \\
& C_{i, j}+r_{\rho_{i, j}, \rho_{i^{\prime}, j^{\prime}}} \leq B_{i^{\prime}, j^{\prime}} \| C_{i^{\prime}, j^{\prime}}+r_{\rho_{i^{\prime}, j^{\prime}, \rho_{i, j}}} \leq B_{i, j} \\
& \forall i, i^{\prime} \in\{1,2, \ldots, n\}, \forall j \in\left\{1,2, \ldots,\left|\rho_{i}\right|\right\}, \forall j^{\prime} \in\left\{1,2, \ldots,\left|\rho_{i^{\prime}}\right|\right\}, \varphi_{\rho_{i, j}}=\varphi_{\rho_{i^{\prime}, j^{\prime}}}
\end{aligned}
$$


sequence of job $i, B_{i, j} \geq 0$.

$C_{i, j}$ Continuous variable for the completion time of the $\mathrm{jth}$ operation in the operation sequence of job $i, C_{i, j} \geq 0$.

$T_{i}$ Continuous variable for tardiness of job $i$, $T_{i} \geq 0$.

$X_{k}$ Continuous variable for position of machine $\mathrm{k}$ on X-coordinate, $X_{k} \geq 0$.

$Y_{k}$ Continuous variable for position of machine $\mathrm{k}$ on Y-coordinate, $Y_{k} \geq 0$.

$\Delta X_{k, k}$, Continuous variable for the distance between machine $\mathrm{k}$ and machine $k$ ' on X-coordinate, $\Delta X_{k, k}, \geq 0$.

$\Delta Y k, k$ ' Continuous variable for the distance between machine $\mathrm{k}$ and machine $\mathrm{k}^{\prime}$ on Y-coordinate, $\Delta Y_{k, k}, \geq 0$.

These decision variables subject to the following constraints:

Constraint (1) describes the completion time of the jth operation in the operation sequence of job i. Constraint (2) defines the tardiness of job $i$. Constraints (3) and (4) respectively calculate the distance between a pair of machines on the $\mathrm{X}$ and $\mathrm{Y}$-coor- dinate. Constraint (5) restricts the distance between two machines on the $\mathrm{X}$ or $\mathrm{Y}$-coordinate to be larger or equal to the sum of the security distance of two machines on that axis. Constraints (6) and (7) restrict the feasibility of the beginning time. For two consecutive operations in a job, the beginning time of the following operation cannot be earlier than the sum of the completion time of the preceding operation, the reconfiguration time, and the transportation time. If these two operations are executed on the same machine, the WIP will not shift. If not, the reconfiguration time is equal to 0 . For any two operations accomplished on the same machine, the later operation ought to start after the processing of the former operation and the reconfiguration are finished.

\subsection{Small numerical experiment in CPLEX}

This model is tested with a small numerical experiment resolved in ILOG CPLEX Optimization Studio developed by IBM. The version used is V12.10.0.

In this experiment, there are six jobs, five opera-

Table 1. The process plan for each job in the small numerical experiment

\begin{tabular}{|c|c|c|c|c|c|c|c|}
\hline \multirow{2}{*}{$\frac{\text { Job ID }}{\text { Job1 }}$} & \multirow{2}{*}{$\frac{\text { Due date }}{40}$} & \multicolumn{2}{|l|}{ Unit penalty } & \multicolumn{4}{|c|}{ Operation in sequence /Processing time } \\
\hline & & 1 & Op1/1 & Op2/2 & $\mathrm{Op} 3 / 3$ & Op4/4 & Op5/5 \\
\hline Job2 & 60 & 2 & Op5/1 & Op4/2 & $\mathrm{Op} 3 / 3$ & Op2/4 & Op $1 / 5$ \\
\hline Job3 & 10 & 3 & Op1/2 & $\mathrm{Op} 3 / 4$ & Op5/1 & $\mathrm{Op} 2 / 3$ & Op4/5 \\
\hline Job4 & 30 & 4 & Op5/4 & $\mathrm{Op3} / 2$ & Op1/5 & Op $4 / 3$ & Op2/1 \\
\hline Job5 & 20 & 5 & Op2/1 & Op4/5 & Op5/4 & Op3/2 & Op $1 / 3$ \\
\hline Job6 & 50 & 6 & Op $4 / 3$ & Op2/2 & Op1/4 & Op3/5 & Op5/1 \\
\hline
\end{tabular}

Table 2. The reconfiguration time in the small numerical experiment

\begin{tabular}{llllll}
\hline Operation ID & Op1 & Op2 & Op3 & Op4 & Op5 \\
\hline Op1 & 0 & 0 & 0 & 0 & 2 \\
Op2 & 0 & 0 & 0 & 0 & 0 \\
Op3 & 0 & 0 & 0 & 0 & 0 \\
Op4 & 0 & 0 & 0 & 0 & 0 \\
Op5 & 4 & 0 & 0 & 0 & 0 \\
\hline
\end{tabular}


tions, and four machines. Jobs due dates, and related unit tardiness time penalty, and the process plans including the operation sequences for jobs and the processing time for every operation are given in Table 1. All time related measurements are in the same time unit.

The security distances of each machine on $\mathrm{X}$ and Y-coordinates are as follows: Machine1 (1,1), Machine2 (2,2), Machine3 (3,3) and Machine4 $(4,4)$. Machine 1 is capable of implementing Op1 and Op5. Op2, Op3, and Op4 are implemented severally on Machine 2, Machine 3, and Machine 4. Table 2 lists the reconfiguration time.

The exact optimal scheduling for jobs and on machines are presented in Figure 2 and Figure 3, respectively. Details are delineated in Table 3 . The optimal layout for this small case is illustrated in Figure 4. From this set of the exact solution, solely Job 2 and Job 6 will be fulfilled on time. The tardiness for Job 1, Job 3, Job 4, and Job 5 is 24, 34, 7, and 18, respectively, giving rise to the minimum penalty of 244 .

\section{Heuristic approach}

Though the CPLEX Optimizer has the high performance to solve the linear programming problem, it is time-consuming to find the exact global optimum for an NP-hard problem. Genetic algorithms have been successfully adopted to solve the flexible jobshop scheduling problem [27]. An improved genetic algorithm is put forward to find the approximate optimal solution within the acceptable time.

Table 3. The beginning time of each operation for each job in the small numerical experiment obtained in CPLEX

\begin{tabular}{|c|c|c|c|c|c|}
\hline \multirow{2}{*}{$\begin{array}{l}\text { Job ID } \\
\text { Job1 }\end{array}$} & \multicolumn{5}{|c|}{ Operation in sequence /The beginning time } \\
\hline & Op1/11 & Op2/16 & Op3/26 & Op4/44 & Op5/59 \\
\hline Job2 & Op5/4 & Op4/13 & Op3/31 & Op2/42 & Op1/52 \\
\hline Job3 & Op1/9 & Op3/15 & Op5/25 & Op2/30 & Op $4 / 39$ \\
\hline Job4 & Op5/0 & Op3/8 & Op1/14 & Op4/27 & Op2/36 \\
\hline Job5 & Op2/1 & Op4/8 & Op5/21 & Op3/29 & Op1/35 \\
\hline Job6 & Op $4 / 0$ & Op2/18 & Op1/30 & Op3/38 & Op5/47 \\
\hline
\end{tabular}

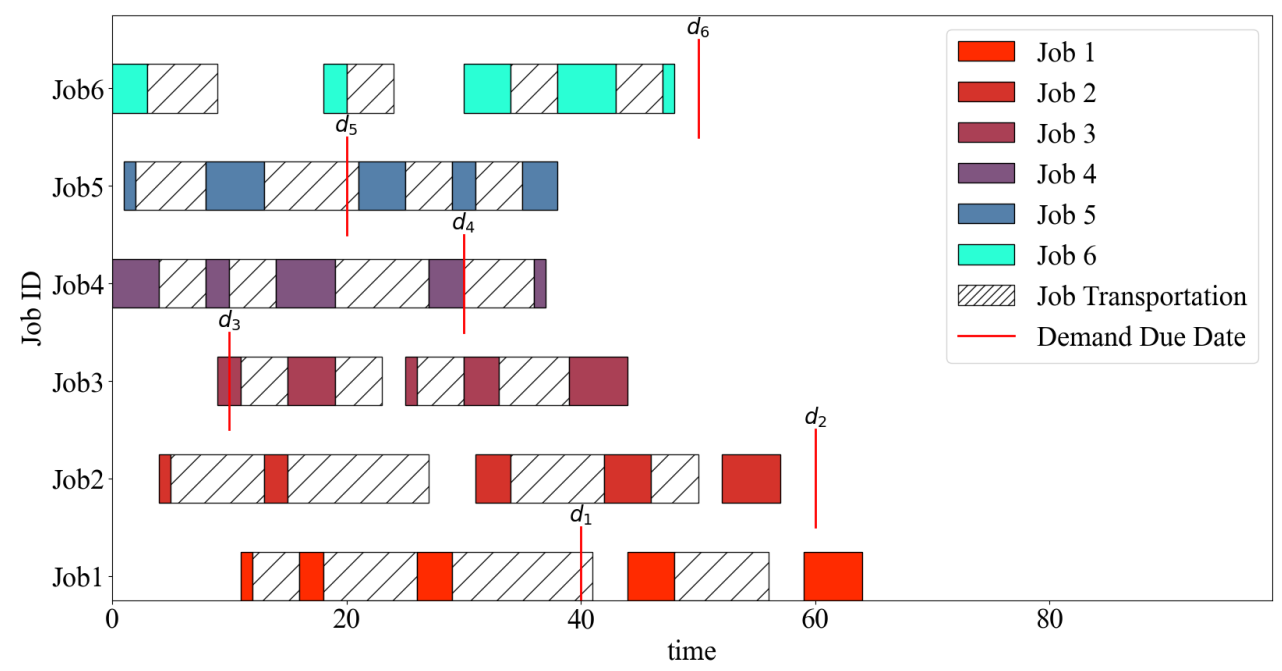

Figure 2. The optimal scheduling for jobs in the small numerical experiment obtained in CPLEX 


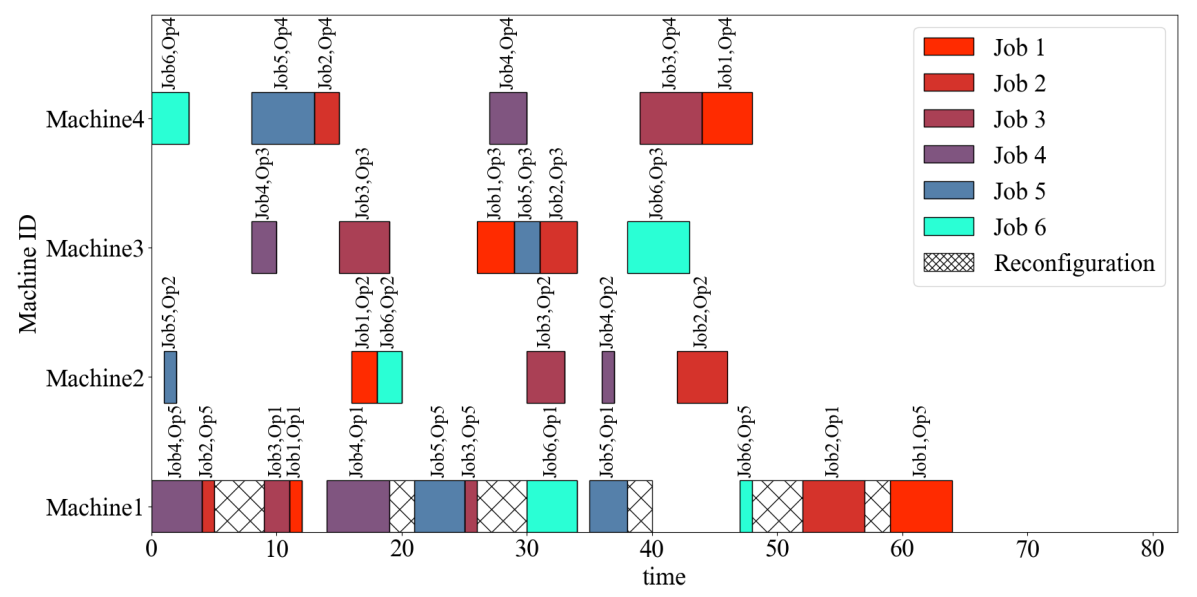

Figure 3. The optimal scheduling on machines in the small numerical experiment obtained in CPLEX

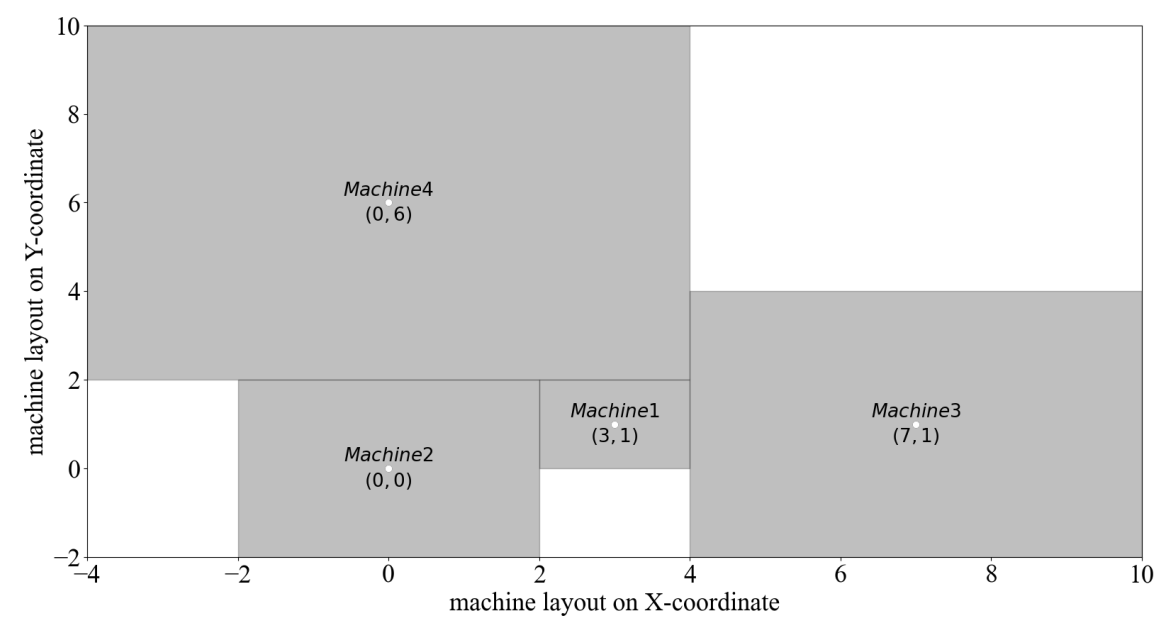

Figure 4. The optimal layout for the small numerical experiment obtained in CPLEX

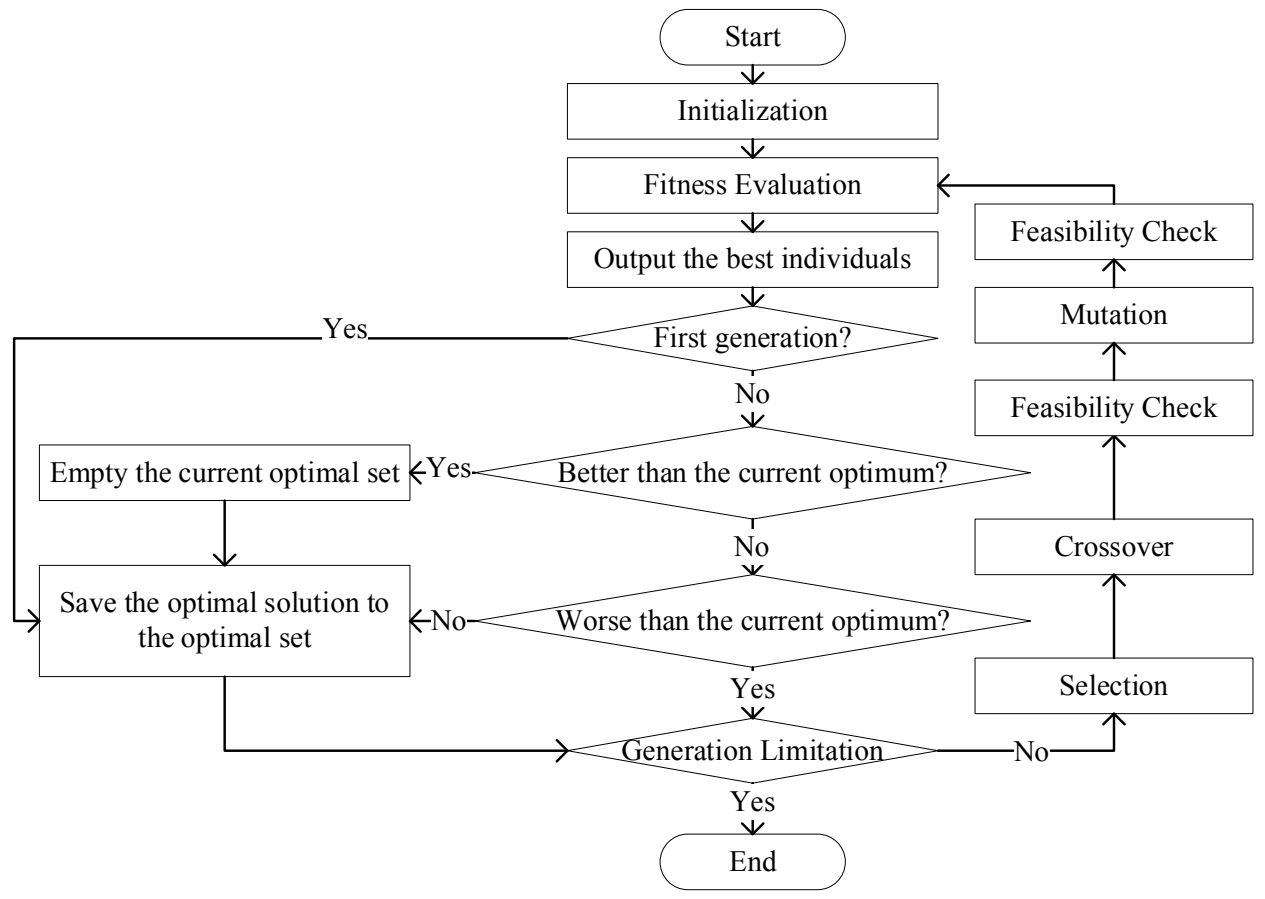

Figure 5. The procedure of the improved GA 


\subsection{Approach presentation}

The primary procedure for this approach is outlined in Figure 5. The best solutions in every generation will be conditionally archived in an optimal set. This helps to save the optimal solutions destroyed by the crossover or mutation operator during iterations. Besides, it can record a couple of optimal solutions having the same optimal objective function value.

Solutions modified by mutation and crossover operators are possibly unfeasible. To comply with the Constraint (5), a technique for repairing the decision variables $\mathrm{X}_{\mathrm{k}}$ and $\mathrm{Y}_{\mathrm{k}}$ is demonstrated as follows:

Layout check Algorithm :

Step 1: $\quad$ Sort all machines in ascending order of their position values on the $\mathrm{X}$-coordinate;

Step 2: $\quad$ Start from the first machine in the order above:

Set this machine as the current machine;

Step 3: $\quad$ Start from the next machine in the above order :

Set this machine as the selected machine;

Step 4: Calculate the distances between the selected machine and the current machine on the $\mathrm{X}$ and Y-coordinate;

Step 5: If the distances do not meet the constraint (5):

Set the position value of the selected machine on the $\mathrm{X}$-coordinate equal to the sum of the position value of the current machine on the $\mathrm{X}$-coordinate and the security distance of these two machines;

Else:

Continue;

Step 6: Sort all machines in ascending order of their position values on the Y-coordinate;

Step 7: $\quad$ Start from the first machine in the order above:

Set this machine as the current machine;

Step 8: $\quad$ Start from the next machine in the above order :

Set this machine as the selected machine;

Step $9 \quad$ Calculate the distances between the selected machine and the current machine on the $\mathrm{X}$ and Y-coordinate;

Step 10: If the distances do not meet the constraint (5):

Set the position value of the selected machine on the Y-coordinate equal to the sum of the position value of the current machine on the $\mathrm{X}$-coordinate and the security distance of these two machines;

Else:

Continue;

End.

To comply with the Constraint (6) and the Constraint (7), a technique for repairing the decision variables $B_{i, j}$ is demonstrated as follows:

Scheduling check Algorithm :

Step 1: $\quad$ Start from the first job:

Start from the first operation in this job operation sequence:

Set this operation as the current operation;

Step 2: If the beginning time of the next operation does not meet the Constraint (6):

Set the beginning time of the next operation equal to the beginning time of the current operation plus the transportation time and the reconfiguration time;

Else:

Continue;

Step 3: $\quad$ Count the total operations of all jobs and set this number as $\mathrm{Opn}$;

Step 4: $\quad$ While $i<O p n$ :

Step 5: $\quad$ Find the minimum beginning time of the remaining operations and set it as the current operation;

Step 6: If the current operation is the first operation in a job operation sequence and the first operation on a machine:

Step 7: Set the beginning time of the current operation equal to 0 ;

Step 8: If the current operation is the first operation in a job operation sequence but not the first operation on a machine: 
Step 9: Set the beginning time of the current operation equal to the completion time of the previous operation on this machine plus the reconfiguration time;

Step 10: If the current operation is not the first operation in a job operation sequence but the first operation on a machine:

Step 11: Set the beginning time of the current operation equal to the completion time of the previous operation in this job operation sequence plus the transportation time;

Step 12: Else:

Step 13: Set the beginning time of the current operation equal to the maximum of the completion time of the previous operation on this machine plus the reconfiguration time and the completion time of the previous operation in that job operation sequence plus the transportation time;

Step 14: $\quad$ End.

\subsection{GA performance validation}

This approach is programmed by Python language in the Integrated Development Environment (IDE) of Pycharm. The version used is 2020.3.

It is first tested with the same small numerical experiment described in section 3.3. Figures 6 and 7 depict one set of the approximate optimal scheduling

Table 4. The beginning time of each operation for each job in the small numerical experiment obtained by the improved GA

\begin{tabular}{|c|c|c|c|c|c|}
\hline \multirow{2}{*}{$\begin{array}{l}\text { Job ID } \\
\text { Job1 }\end{array}$} & \multicolumn{5}{|c|}{ Operation in sequence /The beginning time } \\
\hline & Op1/ 11 & Op2/ 15 & Op3/ 24 & Op4/ 43 & Op5/ 59 \\
\hline Job2 & Op5/ 4 & Op4/ 14 & Op3/ 31 & Op2/ 41 & Op1/ 52 \\
\hline Job3 & Op1/ 9 & Op3/ 15 & Op5/ 25 & Op2/ 29 & Op4/ 38 \\
\hline Job4 & Op5/0 & Op3/8 & Op1/14 & Op4/28 & Op2/37 \\
\hline Job5 & Op2/0 & Op4/7 & Op5/21 & Op3/29 & Op1/35 \\
\hline Job6 & Op4/0 & Op2/9 & Op1/30 & Op3/38 & Op5/47 \\
\hline
\end{tabular}

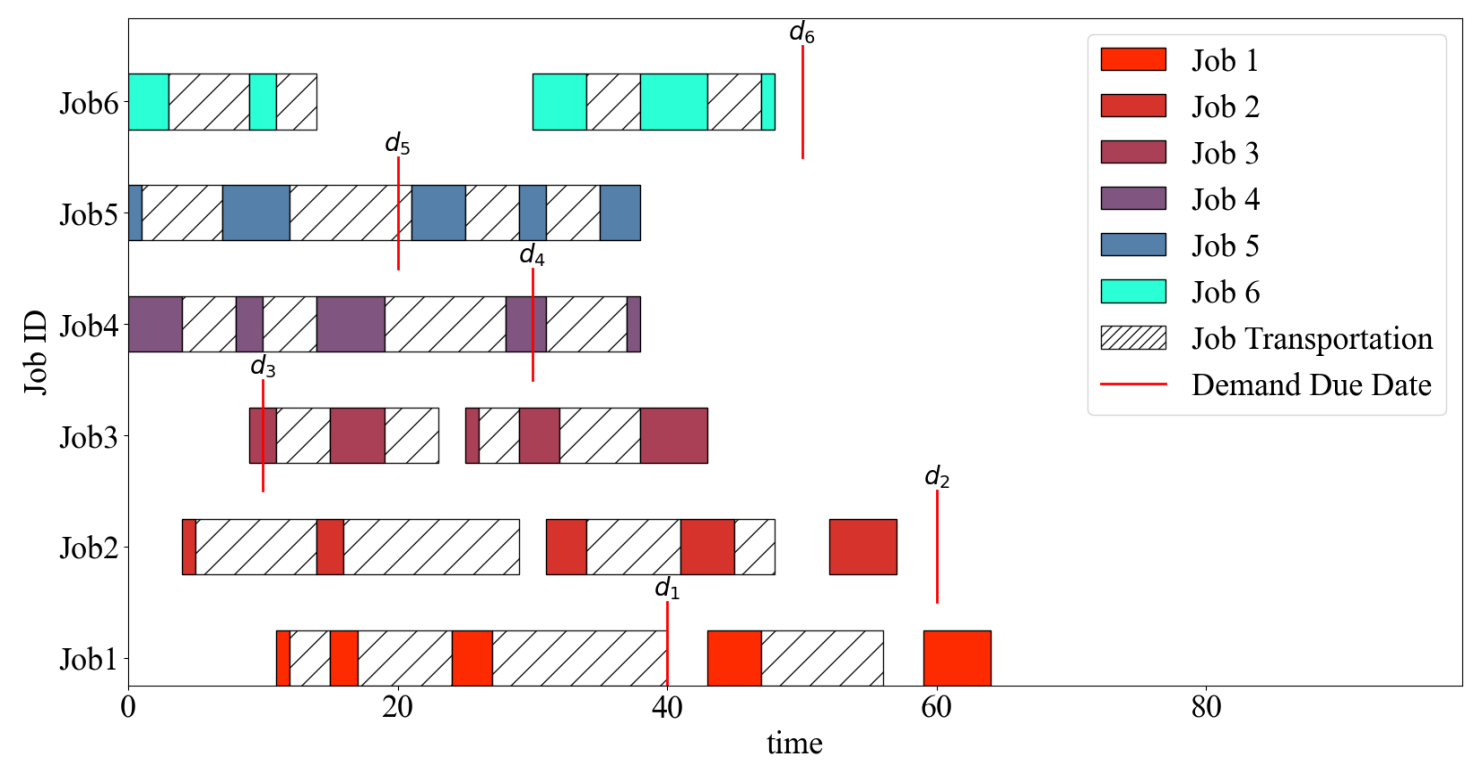

Figure 6. The optimal scheduling for jobs in the small numerical experiment obtained by the improved GA 
for jobs and on machines. The accurate values for the beginning time of operations are mapped out in Table 4. Similar to the exact optimal solutions, Job 2 and Job 6 will finish on time. The tardiness for Job 1, Job 3 , Job 4, and Job 5 is 24, 33, 8, and 18, respectively, inducing a penalty of 245 . The approximate optimal layout derived from this approach is illustrated in Figure 8. Despite the difference in machines positions, the structure is similar to that appointed in the exact optimal solution.

\section{Discussion}

The computation was conducted in a laptop computer powered by an Intel Core i7-7600U CPU (2.80 $\mathrm{GHz}$ ) and $16 \mathrm{~GB}$ of RAM. By running the small numerical experiment ten times in CPLEX, the computation time (C_t $)$ of each run is presented in Table 5. The average computation time to obtain the exact optimal solution in CPLEX is 27.84 seconds.

The performance of the improved GA depends on the generation limit (g), the population size (s),

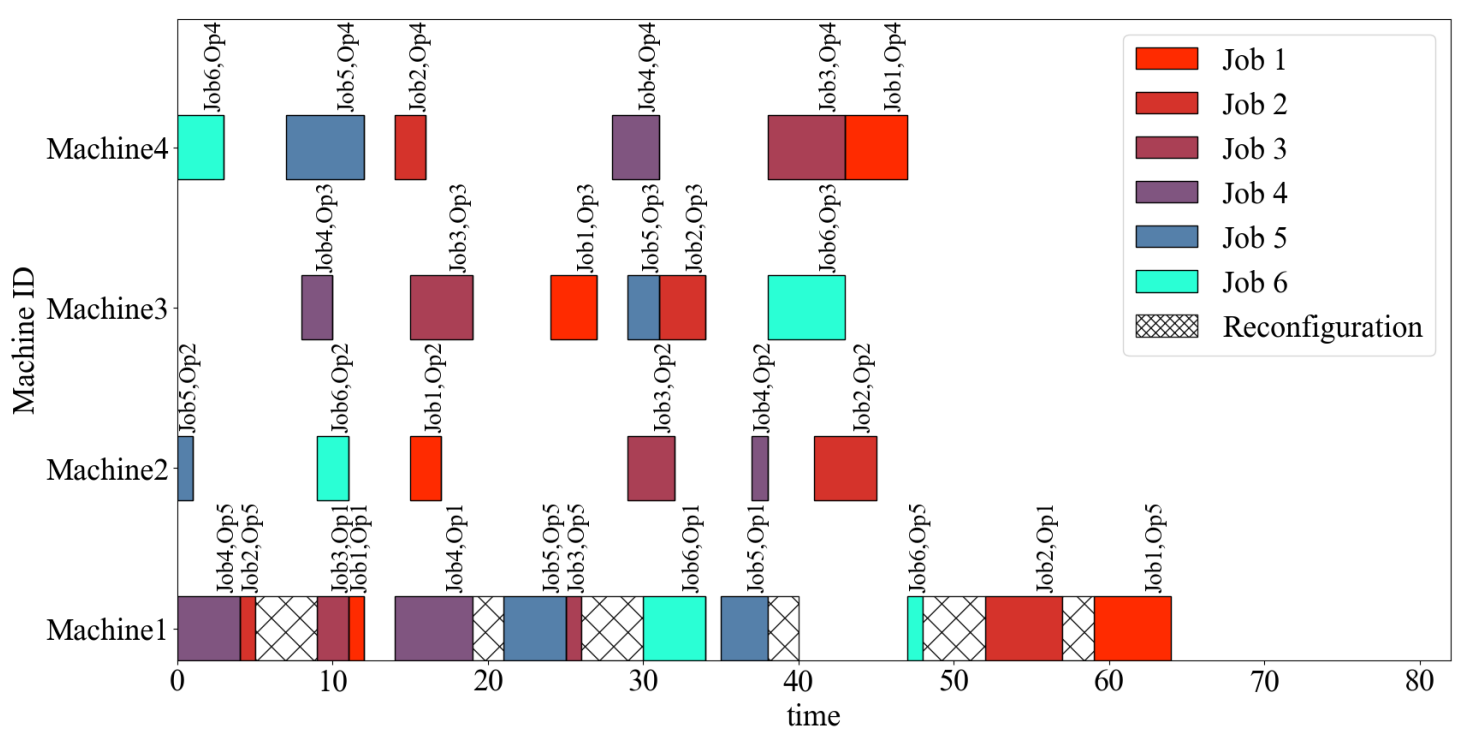

Figure 7. The optimal scheduling on machines in the small numerical experiment obtained by the improved GA

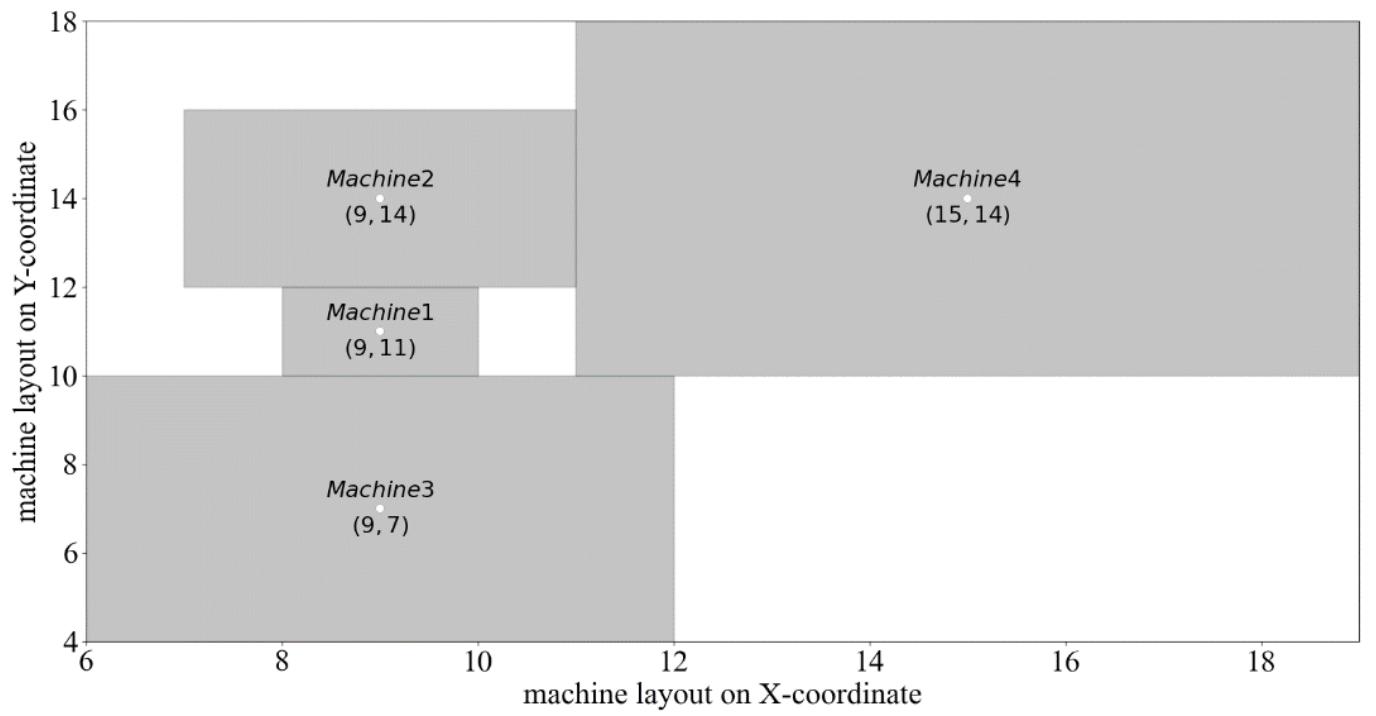

Figure 8. The optimal layout for the small numerical experiment obtained by the improved GA 
and the mutation probability (p_m). By running the small numerical experiment ten times separately with four sets of parameters for the improved GA, the computation time (t_v), objective function value, and deviation are obtained and are shown in Table 5 . The deviation to assess the approximate optimal solution obtained by the improved GA for the small numerical experiment is defined as follows:

$$
d_{v}=\frac{\left|f_{v}-f_{o p t}\right|}{f_{v}}
$$

$d_{v}$ Deviation between the objective value obtained in the $v^{\text {th }}$ run and the objective value of the exact optimal solution

$f_{v} \quad$ The objective value obtained in the $v^{t h}$ run

$f_{\text {opt }}$ The objective value of the exact optimal solution

The generation limit and population size obviously influence the computation time and the distribution of the approximate solutions. Despite the increase in computing time, a large population enhances the accuracy of the approximate solutions, whereas the excessive iterations do not improve the performance. Mutation probability affects the computation time little while having a big impact on the deviation of the approximate solutions.

In fact, the exact solutions for the optimal lay- out are numerous because there is no boundary for the workshop. Compared with the performance of CPLEX, the improved GA could get several approximate solutions that are close to the exact solutions within a short time. With optimal parameters tuning, this approach may obtain optimal solution.

\section{Conclusion}

This paper presented a linear mathematical model to integrate the job shop scheduling problem with the layout configuration in an RMS. It answered the following questions:

(1) In what order to process these operations;

(2) When is the processing beginning time for these operations;

(3) What is the optimal RMS layout.

The process plan is deterministic. The single objective is to minimize the total penalty for tardiness. A small-scale example is first implemented in the CPLEX to validate this model. Then it is used to prove the effectiveness and efficiency of the improved GA approach. The numerical experiments are conducted to investigate the relationship between the parameters and the performance of this approach. The limits of this work are in the mathematical model:

Table 5. The performance to solve the small numerical experiment

\begin{tabular}{|c|c|c|c|c|c|c|c|c|c|c|c|c|c|}
\hline \multirow{2}{*}{ Run } & \multirow{2}{*}{$C_{t / s}$} & \multicolumn{3}{|c|}{$\begin{array}{c}g=400, s=40 \\
p_{m}=0.1\end{array}$} & \multicolumn{3}{|c|}{$\begin{array}{c}g=800, s=40 \\
p_{m}=0.1\end{array}$} & \multicolumn{3}{|c|}{$\begin{array}{c}g=400, s=80 \\
p_{m}=0.1\end{array}$} & \multicolumn{3}{|c|}{$\begin{array}{c}g=400, s=40 \\
p_{m}=0.05\end{array}$} \\
\hline & & $t_{v / s}$ & $f_{v}$ & $d_{v}(\%)$ & $t_{v / s}$ & $f_{v}$ & $d_{v}(\%)$ & $t_{v / s}$ & $f_{v}$ & $\mathrm{~d}_{\mathrm{v}}(\%)$ & $\mathrm{t}_{\mathrm{v} / \mathrm{s}}$ & $f_{v}$ & $\mathrm{~d}_{\mathrm{v}}(\%)$ \\
\hline 1 & 25.86 & 6.84 & 244 & $0.00 \%$ & 12.61 & 245 & $0.41 \%$ & 12.95 & 245 & $0.41 \%$ & 5.84 & 261 & $6.51 \%$ \\
\hline 2 & 27.34 & 6.72 & 245 & $0.41 \%$ & 12.52 & 244 & $0.00 \%$ & 12.84 & 245 & $0.41 \%$ & 6.47 & 269 & $9.29 \%$ \\
\hline 3 & 27.35 & 6.39 & 255 & $4.31 \%$ & 12.72 & 264 & $7.58 \%$ & 14.31 & 244 & $0.00 \%$ & 5.86 & 255 & $4.31 \%$ \\
\hline 4 & 28.66 & 6.56 & 245 & $0.41 \%$ & 13.98 & 261 & $6.51 \%$ & 13.70 & 245 & $0.41 \%$ & 6.55 & 245 & $0.41 \%$ \\
\hline 5 & 28.63 & 6.55 & 245 & $0.41 \%$ & 12.48 & 255 & $4.31 \%$ & 12.95 & 245 & $0.41 \%$ & 6.53 & 245 & $0.41 \%$ \\
\hline 6 & 28.95 & 7.23 & 256 & $4.69 \%$ & 12.86 & 245 & $0.41 \%$ & 12.84 & 244 & $0.00 \%$ & 5.86 & 261 & $6.51 \%$ \\
\hline 7 & 28.52 & 6.48 & 245 & $0.41 \%$ & 14.09 & 245 & $0.41 \%$ & 14.16 & 249 & $2.01 \%$ & 5.81 & 246 & $0.81 \%$ \\
\hline 8 & 27.74 & 7.17 & 249 & $2.01 \%$ & 12.44 & 245 & $0.41 \%$ & 12.95 & 244 & $0.00 \%$ & 6.55 & 245 & $0.41 \%$ \\
\hline 9 & 27.59 & 6.91 & 246 & $0.81 \%$ & 12.56 & 259 & $5.79 \%$ & 13.13 & 244 & $0.00 \%$ & 5.88 & 260 & $6.15 \%$ \\
\hline 10 & 27.76 & 6.50 & 261 & $6.51 \%$ & 14.03 & 249 & $2.01 \%$ & 13.02 & 245 & $0.41 \%$ & 5.95 & 255 & $4.31 \%$ \\
\hline Mean & 27.84 & 6.735 & 249.1 & $2.00 \%$ & 13.029 & 251.2 & $2.78 \%$ & 13.285 & 245 & $0.40 \%$ & 6.13 & 254.2 & $3.91 \%$ \\
\hline
\end{tabular}


(1) An operation could be processed on several configurations;

(2) The setup time should be accounted;

(3) The workshop has finite space.

The contribution of this work is advancing an efficient method to optimize the scheduling and layout simultaneously in an RMS. This is significant based on the flexibility of RMS but rare researchers work on it. The way to use the continuous variables to decide the beginning time of operations will decrease the number of the independent decision variables and easy for some powerful business solvers to calculate. Techniques proposed in this paper to modify the infeasible solutions help to quickly find the optimum solution. . Compared with the method proposed by E. S. Hernández-Gress [28] to concurrently solve the scheduling and layout problems, this paper considers an open-field layout which is more possible in an RMS environment. However, the reconfiguration time depends on the processing sequence on machines, complicating the scheduling by influencing the makespan. Future work aims at integrating process planning, scheduling, and layout configuration to produce mass customized products in RMS. Also, it aims at overcoming this work limits. In addition to tardiness, cost and energy consumption can be minimized in a multi-objective model. The performance of the GA based approach can be improved with parameters tuning. The P-Modules ideas proposed by Chatzopoulos [29] could be adopted to satisfy the dynamic demands for mass customized products in RMS.

\section{Funding}

This work was supported by China Scholarship Council (CSC) [contract number 201806280501].

\section{References}

[1] E. Sandrin, A. Trentin, and C. Forza, "Organizing for mass customization: Literature review and research Agenda," Int. J. Ind. Eng. Manag., vol. 5, no. 4, pp. 159-167, 2014.

[2] G. Da Silveira, D. Borenstein, and F. S. Fogliatto, "Mass customization: Literature review and research directions," Int. J. Prod. Econ., vol. 72, no. 1, pp. 1-13, 2001, doi: 10.1016/S0925-5273(00)00079-7.

[3] K. Dorofeev, S. Profanter, J. Cabral, P. Ferreira, and A. Zoitl, "Agile Operational Behavior for the Control-Level Devices in Plug\&Produce Production Environments," IEEE Int. Conf. Emerg. Technol. Fact. Autom. ETFA, vol. 2019-Septe, no. 680735, pp. 49-56, 2019, doi: 10.1109/ ETFA.2019.8869208.

[4] S. N. Joergensen, K. Nielsen, and K. A. Joergensen, "Re configurable manufacturing systems as an application of mass customisation,” Int. J. Ind. Eng. Manag., vol. 1, no. 3, pp. 111-119, 2010.

[5] H. Haddou, "A New Hybrid Approach for Machine Layout Design Under Family Product Evolution for Reconfigurable Manufacturing Systems," IFAC Pap., vol. 52, no. 13, pp. 1379-1384, 2019, doi: 10.1016/ j.ifacol.2019.11.391.

[6] Y. Koren, U.Heisel, F. Jovane, T. Moriwaki, G. Pritschow, G. Ulsoy, H.Van Brussel, "Reconfigurable manufacturing systems," in CIRP Annals - Manufacturing Technology, 1999, vol. 48, no. 2, pp. 527-540. doi: 10.1016/ S0007-8506(07)63232-6.

[7] A. Bensmaine, M. Dahane, and L. Benyoucef, "A nondominated sorting genetic algorithm based approach for optimal machines selection in reconfigurable manufacturing environment," Comput. Ind. Eng., vol. 66, no. 3, pp. 519-524, 2013, doi: 10.1016/j.cie.2012.09.008.

[8] R. C. Sabioni, J. Daaboul, and J. Le Duigou, "An integrated approach to optimize the configuration of mass-customized products and reconfigurable manufacturing systems," Int. J. Adv. Manuf. Technol., pp. 141-163, 2021, doi: 10.1007/s00170-021-06984-w.

[9] X. Li, A. E. Bayrak, B. I. Epureanu, and Y. Koren, "Real-time teaming of multiple reconfigurable manufacturing systems," CIRP Ann., vol. 67, no. 1, pp. 437-440, 2018, doi: 10.1016/j.cirp.2018.04.051.

[10] M. Mahmoodjanloo, R. Tavakkoli-Moghaddam, A. Baboli, and A. Bozorgi-Amiri, "Flexible job shop scheduling problem with reconfigurable machine tools: An improved differential evolution algorithm,” Appl. Soft Comput. J., vol. 94, p. 106416, 2020, doi: 10.1016/j.asoc.2020.106416.

[11] V. Roshanaei, A. Azab, and H. Elmaraghy, "Mathematical modelling and a meta-heuristic for flexible job shop scheduling," Int. J. Prod. Res., vol. 51, no. 20, pp. 6247-6274, 2013, doi: 10.1080/00207543.2013.827806.

[12] A. Azab and B. Naderi, "Modelling the problem of production scheduling for reconfigurable manufacturing systems," Procedia CIRP, vol. 33, pp. 76-80, 2015, doi: 10.1016/j.procir.2015.06.015.

[13] M. Abbasi and M. Houshmand, "Production planning and performance optimization of reconfigurable manufacturing systems using genetic algorithm," Int. J. Adv. Manuf. Technol., vol. 54, no. 1-4, pp. 373-392, 2011, doi: 10.1007/ s00170-010-2914-x.

[14] A. Bhargav, C. N. V. Sridhar, and M. L. S. Deva Kumar, "Study of Production Scheduling Problem for Reconfigurable Manufacturing System (RMS),” Mater. Today Proc., vol. 4, no. 8, pp. 7406-7412, 2017, doi: 10.1016/j.matpr.2017.07.071.

[15] L. Aiping and X. Nan, "A robust scheduling for reconfigurable manufacturing system using petri nets and genetic algorithm," Proc. World Congr. Intell. Control Autom., vol. 2, no. 031111002, pp. 7302-7306, 2006, doi: 10.1109/WCICA.2006.1714504.

[16] P. Ladosz, O. Banjo, S. De Guido, and M. Zalasiewicz, "A Genetic Algorithm Optimiser for Dynamic Product Routing in Agile Manufacturing Environment," Proc. - IEEE 16th Int. Conf. Ind. Informatics, INDIN 2018, pp. 1079-1084, 2018, doi: 10.1109/INDIN.2018.8472089.

[17] H. Ye and M. Liang, "Solving the combined modular product scheduling and production cell reconfiguration problem: A GA approach with parallel chromosome coding," 2005 IEEE Congr. Evol. Comput. IEEE CEC 2005. Proc., vol. 2, pp. 1348-1355, 2005, doi: 10.1109/ cec.2005.1554847.

[18] J. Dou, J. Li, D. Xia, and X. Zhao, "A multi-objective particle swarm optimisation for integrated configuration design and scheduling in reconfigurable manufacturing system,” Int. J. Prod. Res., vol. 0, no. 0, pp. 1-21, 2020, doi: 10.1080/00207543.2020.1756507. 
[19] A. Khezri, H. H. Benderbal, and L. Benyoucef, "A Sustainable Reconfigurable Manufacturing System Designing with Focus on Environmental Hazardous Wastes," IEEE Int. Conf. Emerg. Technol. Fact. Autom. ETFA, vol. 2019-Septe, pp. 317-324, 2019, doi: 10.1109/ ETFA.2019.8869380.

[20] I. Maganha, C. Silva, and L. M. D. F. Ferreira, "The layout design in reconfigurable manufacturing systems: a literature review," Int. J. Adv. Manuf. Technol., vol. 105, no. 1-4, pp. 683-700, 2019, doi: 10.1007/s00170-01904190-3.

[21] S. E. H. Petroodi, A. B. D. Eynaud, N. Klement, R. Tavakkoli-Moghaddam, "Simulation-based optimization approach with scenario-based product sequence in a re configurable manufacturing system (RMS): A case study," IFAC-PapersOnLine., vol. 52, no. 13, pp. 2638-2643, 2019, doi: 10.1016/j.ifacol.2019.11.605.

[22] M. Bruccoleri, G. Lo Nigro, G. Perrone, P. Renna, and S. Noto La Diega, "Production planning in reconfigurable enterprises and reconfigurable production systems," CIRP Ann. - Manuf. Technol., vol. 54, no. 1, pp. 433-436, 2005, doi: 10.1016/S0007-8506(07)60138-3.

[23] H. Haddou Benderbal and L. Benyoucef, "Machine layout design problem under product family evolution in reconfigurable manufacturing environment: a two-phasebased AMOSA approach,” Int. J. Adv. Manuf. Technol., vol. 104, no. 1-4, pp. 375-389, 2019, doi: 10.1007/s00170019-03865-1.

[24] X. Wei, S. Yuan, and Y. Ye, "Optimizing facility layout planning for reconfigurable manufacturing system based on chaos genetic algorithm," Prod. Manuf. Res., vol. 7, no. 1, pp. 109-124, 2019, doi: 10.1080/21693277.2019.1602486.

[25] Z. Sui, T. Hou, L. Duan, and T. Wu, "Research on optimization of production workshop layout based on extension particle swarm optimization," Chinese Control Conf. CCC, vol. 2019-July, no. 1, pp. 2228-2233, 2019, doi: 10.23919/ChiCC.2019.8865315.

[26] Y. Yamada, K. Ookoudo, and Y. Komura, "Layout Optimization of Manufacturing Cells and Allocation Optimization of Transport Robots in Reconfigurable Manufacturing Systems Using Particle Swarm Optimization," IEEE Int. Conf. Intell. Robot. Syst., vol. 2, no. October, pp. 2049-2054, 2003, doi: 10.1109/ iros.2003.1248968.

[27] F. Pezzella, G. Morganti, and G. Ciaschetti, "A genetic algorithm for the Flexible Job-shop Scheduling Problem," Comput. Oper. Res., vol. 35, no. 10, pp. 3202-3212, 2008, doi: 10.1016/j.cor.2007.02.014.

[28] E. S. Hernández-Gress, J. C. Seck-Tuoh-Mora, N. Hernández-Romero, J. Medina-Marín, P. LagosEulogio, and J. Ortíz-Perea, "The solution of the concurrent layout scheduling problem in the job-shop environment through a local neighborhood search algorithm," Expert Syst. Appl., vol. 144, 2020, doi: 10.1016/j.eswa.2019.113096.

[29] C. G. Chatzopoulos, "Flow customizer: An algorithm to design lean-flow production systems for mass customization,” Int. J. Ind. Eng. Manag., vol. 5, no. 4, pp. 179-184, 2014. 